\title{
Effects of a multimodal intervention on gait and balance of subjects with progressive multiple sclerosis: a prospective longitudinal pilot study
}

This article was published in the following Dove Press journal:

Degenerative Neurological and Neuromuscular Disease

26 June 2017

Number of times this article has been viewed

\author{
Babita Bisht ${ }^{1,2}$ \\ Warren G Darling ${ }^{2}$ \\ Emily C White ${ }^{2}$ \\ Kaitlin A White ${ }^{2}$ \\ E Torage Shivapour ${ }^{3}$ \\ $M$ Bridget Zimmerman ${ }^{4}$ \\ Terry L Wahls ${ }^{1,5}$ \\ 'Department of Internal \\ Medicine, Carver College of \\ Medicine, ${ }^{2}$ Department of Health and \\ Human Physiology, UI College of Liberal \\ Arts and Sciences, ${ }^{3}$ Department of \\ Neurology, Carver College of Medicine, \\ ${ }^{4}$ Department of Biostatistics, College \\ of Public Health, University of lowa, \\ ${ }^{5}$ Department of Extended Care and \\ Rehabilitation Service Line, lowa City VA \\ Health Care System, lowa City, IA, USA
}

\section{Video abstract}

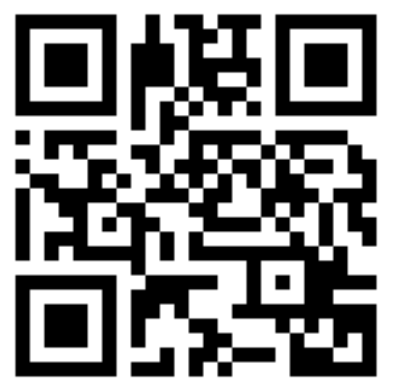

Point your SmartPhone at the code above. If you have a QR code reader the video abstract will appear. Or use: http://youtu.be//sZquj9Drlc

Correspondence: Terry L Wahls Department of Internal Medicine, Carver College of Medicine, University of lowa, SE 629 GH, 200 Hawkins Drive, lowa City, IA 52246, USA

Tel + I 319356442 I

Email terry-wahls@uiowa.edu
Purpose: To investigate the effects of a multimodal intervention including a modified Paleolithic diet, nutritional supplements, stretching, strengthening exercises with electrical stimulation of trunk and lower limb muscles, meditation and massage on walking performance and balance of subjects with progressive multiple sclerosis (MS).

Materials and methods: Twenty subjects with mean (standard deviation) age of 51.7 (6.4) years and Expanded Disability Status Scale score of 6.2 (1) participated in a 12-month study. Assessments were completed at baseline, 3, 6, 9, and 12 months.

Results: The entire cohort did not show significant changes in any of the assessments over 12 months except higher speed of walking toward the 10 feet mark during timed up and go (TUG) test at 6 months compared with baseline (mean change $7.9 \mathrm{~cm} / \mathrm{s}[95 \%$ confidence interval $\{\mathrm{CI}\}]$ : $0.3,15.2 ; p=0.041)$. Sub-group analysis revealed that $50 \%$ subjects $(n=10)$ showed decrease in TUG time from baseline to at least 3 of 4 time-points post-intervention and were considered as responders (TUG-Res), the remaining 10 subjects were considered as nonresponders (TUG-NRes). Over 12 months, TUG-Res showed decreased mean TUG time by $31 \%$ (95\% CI: $-52 \%,-2 \%$ ), increased median Berg Balance Scale scores (42 to 47 ), 30\% increase in mean timed 25 -foot walk speed ( $>20 \%$ considered clinically significant) and increased speed of walk toward 10 feet mark during TUG by $11.6 \mathrm{~cm} / \mathrm{s}(95 \% \mathrm{CI}:-3.0,25.9)$ associated with increases in step lengths and decrease in step duration. TUG-NRes showed deterioration in walking ability over 12 months. Comparison of TUG-Res and TUG-NRes showed no significant differences in adherence to intervention but better stride duration and longer step length at baseline for TUG-Res than for TUG-NRes $(p<0.05)$.

Conclusion: A multimodal lifestyle intervention may improve walking performance and balance in subjects with progressive MS who have mild-to-moderate gait impairment, whereas subjects with severe gait impairments may not respond to this intervention. Future trials should assess effects of this intervention in subjects with MS during early stages of the disease.

Keywords: multiple sclerosis, Paleolithic diet, exercise, neuromuscular electrical stimulation, gait, balance

\section{Introduction}

Over $90 \%$ of persons with multiple sclerosis (pwMS) experience walking impairment, which is a major contributor to disability. ${ }^{1}$ Within 15 years of onset of MS, $~ 50 \%$ of pwMS need some form of walking assistance and $10 \%$ become restricted to wheelchair. ${ }^{2}$ A recent study reported that mobility impairment in MS is a large burden to society as it leads to decreased work productivity, early departure from the work force, and decreased health-related quality of life. ${ }^{3}$ 
Several pharmacological and non-pharmacological treatments have been proposed for MS-related gait and balance impairments. In two Phase III clinical trials, a small proportion of subjects ( $35 \%$ and $42.9 \%$ ) showed $~ 25 \%$ increase in walking speed during timed 25 -foot walk (T25FW) test following use of extended-release dalfampridine $10 \mathrm{mg}$ daily for 14 and 9 weeks, respectively., ${ }^{4,5}$ However, with continuous treatment for 12 months, improvement from baseline was maintained only in walking endurance (distance during 2 minute walk test) and not in walking speed. ${ }^{6}$ Two reviews of effects of exercise training indicated beneficial effects of exercise on muscle strength, balance, walking endurance and speed in pwMS. ${ }^{7,8}$ A review by Latimer et al concluded that exercise training improves muscular strength and endurance in pwMS with mild-to-moderate disability. ${ }^{7}$ Another review reported that exercise training was shown to improve balance and gait parameters in pwMS, although this evidence was mainly based on pilot studies. ${ }^{8}$ Electrical stimulation of nerves/muscles or functional electrical stimulation (FES) is another physical modality that has shown some beneficial effects in pwMS. A pilot study in 5 pwMS (3 secondary progressive MS, 2 primary progressive MS; mean Expanded Disability Status Scale [EDSS] scores 6.5, range: 6-6.5) showed that a progressive resistance training program with a FES bike decreased time to complete timed up and go (TUG) test (by $22 \%$ at 6 months from baseline) and T25FW (by $36 \%$ at 6 months from baseline). ${ }^{9}$ However, FES to prevent foot drop while walking has been most commonly studied in pwMS. Like an orthotic device, FES for foot drop can immediately improve walking performance and was shown to reduce falls in pwMS. ${ }^{10}$ However, studies investigating therapeutic effects of FES, that is, effects after stimulation is turned off have shown conflicting results. ${ }^{10,11}$ In a recent review and meta-analysis, Miller et al evaluated studies investigating the effects of FES used for foot drop on gait speed in short and long walking performance tests in pwMS. ${ }^{12}$ Authors reported significant initial orthotic effects of FES on gait speed during short walk tests (mean increase of 0.05 $\mathrm{m} / \mathrm{s}, p=0.016$ ) but not during long walk tests. Additionally, no significant therapeutic effects of FES on gait speed during short or long walk tests were found. Some research has also demonstrated positive effects of stress management programs for individuals with MS. For example, a randomized, controlled trial of a stress management program (relaxation breathing and progressive muscle relaxation, twice a day for 8 weeks) reported significantly reduced number and intensity of perceived MS symptoms compared with control group. ${ }^{13}$ Another randomized controlled trial of stress management therapy for 24 weeks reported reduction in new $\mathrm{Gd}+$ lesions in the intervention group. Moreover, a greater number of subjects in the treatment group remained free of new $\mathrm{Gd}+$ lesions (76.8\% vs 54.7\%) compared with the control group. ${ }^{14}$ Although currently available treatments have shown some beneficial effects, treatments to effectively stop or reverse gait impairment in MS are needed. Some studies suggest that a multimodal approach may be effective in treating MS-related gait impairment. A case report of an individual with secondary progressive MS reported significant improvement in walking ability (scooter dependence to ability to walk without assistance) following combined use of a nutrient-dense diet, nutritional supplements, strengthening exercises, and neuromuscular electrical stimulation (NMES), suggesting that therapeutic effects of exercise and neuromuscular stimulation in MS may be enhanced by an appropriate diet. ${ }^{15} \mathrm{~A}$ retrospective case series reported that following combined use of NMES and a home-based exercise program for 22-495 days, 8 of 9 subjects with progressive MS showed improved gait performance according to physical therapist's observational analysis, with an average decrease of 0.78 point in EDSS scores (range: 0-2.0). ${ }^{16}$ Above mentioned studies provide only a low level of evidence due to small number of subjects and lack of controls. However, robust improvements reported in subjects in progressive phase of MS, when worsening of gait and other MS symptoms is expected, ${ }^{17,18}$ strongly suggest beneficial effects of a multimodal intervention. Thus, we investigated the effects of a multimodal intervention including a modified Paleolithic diet with supplements, stretching, strengthening exercises with electrical stimulation of the trunk and lower limb muscles, and stress management on individuals with secondary and primary progressive MS over a period of 12 months. We hypothesized that a multimodal intervention will result in improved gait and balance in subjects with progressive MS at 3, 6, 9, and 12 months from baseline. We recently reported that this intervention is feasible ${ }^{19}$ and may decrease fatigue and improve quality of life of individuals with progressive MS. ${ }^{20}$

\section{Materials and methods Design overview}

This was a prospective longitudinal Phase I pilot study with no control group. Effects of a multimodal intervention in subjects with progressive MS were assessed over a period of 12 months. Potentially eligible patients with primary and secondary progressive MS from the spinal cord injury clinics at Iowa City VAMC and University of Iowa Hospitals and Clinics (UIHC) neurology department were provided 
information about the study by their doctors (Dr Bingham at VAMC and Dr Shivapour ET, of UIHC Neurology). In addition, eligible patients of Dr Shivapour ET, were sent letters with information regarding the study. Some subjects contacted the study team after they heard about the clinical trial from other resources. Subjects who were interested in participating in the study were asked screening questions to establish eligibility to participate in run-in period of the study. During first study visit, eligible subjects were enrolled into a run-in period and were asked to follow the study diet and a stretching exercise program for 2 weeks. Subjects who followed the study diet for 7 consecutive days during the 2 -week run-in period were enrolled into the 12-month main study at their second (2-week) visit. All assessments were completed at baseline (spread over two run-in visits) and at $3,6,9$, and 12 months into the main study. This study was approved by University of Iowa Institutional Review Board (IRB; study number 201611800) and was conducted according to the principles expressed in the Declaration of Helsinki. Informed written consent was obtained from all participants involved in the study. The study was prospectively registered at ClinicalTrials.gov (NCT01381354).

\section{Setting and participants}

Recruitment of subjects and data collection for this study were performed at the Clinical Research Unit at UIHC. Recruitment of subjects started on October 6, 2010 and 12-month study visit of last subject was completed on December 2, 2013. Subjects with confirmed diagnosis of secondary or primary progressive MS by a neurologist specializing in MS (Shivapour ET), stable medical status in prior 3 months and having at least mild gait disability were enrolled in the study. Exclusion criteria were: abnormal kidney or liver functions, clinically significant cognitive dysfunction, unstable heart or lung disease and any implanted device. Detailed description of inclusion and exclusion criteria were provided previously. ${ }^{19}$ Due to IRB concerns, we were asked to first enroll 10 subjects into the 12-month phase of the study and submit a safety report following 6 months of intervention. Thus, 9 participants were part of a first group attempting the intervention after which the next 11 subjects were recruited following review of a safety report by the University of Iowa IRB.

\section{Intervention}

A multimodal intervention consisting of a modified Paleolithic diet, a home exercise program including stretching and strengthening exercises of trunk and lower limb muscles,
NMES of trunk and lower limb muscles, stress reduction techniques and nutritional supplements, was used. Education regarding study diet was provided by Wahls TL, and about stretching exercises was provided by Bisht $\mathrm{B}$, to subjects and their adult companion during first run-in visit. Subjects and their adult companion were trained on strengthening exercises and use of NMES by Bisht B, and on stress management techniques (meditation and massage) and optional nutritional supplements by Wahls TL, during second study visit. Detailed description of each component of this intervention was provided previously. ${ }^{19,20}$ Briefly, the study diet included leafy green and sulfur-containing vegetables, intensely colored fruits and vegetables, plant and animal protein, seaweed and non-dairy milks and excluded gluten-containing grains, eggs and dairy. Subjects were considered adherent to study diet on a particular day if they consumed any of recommended foods and did not consume any of excluded foods. Stretching and strengthening exercises of lower limb and trunk muscles personalized to each subject were recommended for 5 days/ week. Most strengthening exercises were performed with simultaneous use of NMES to assist with the exercises. Subjects were considered adherent to NMES-exercise program on a particular day if they either performed exercises and/or applied NMES. Stress reduction techniques included self-massage and meditation, and were recommended for 20 minutes per day. To facilitate adherence with study diet, subjects were provided with recipes and menus. Trained research assistants called subjects twice during 2-week run-in period to answer any questions subjects had regarding diet and stretching exercises. After enrollment into main study, research assistants called the subjects weekly in the first 2 months to answer any questions related to the intervention. Subjects were encouraged to call the study team if they had any question about the intervention during the study period. Research assistants used motivational interviewing techniques such as use of open-ended questions and reflective statements while talking to subjects to improve and sustain adherence with intervention.

Subjects were asked to complete daily logs of their food intake, exercises, and duration of electrical stimulation. At enrollment, subjects consented to mail their weekly logs; however, for subject convenience, we let subjects bring the logs with them during their study visits. We provided several nutritional supplements as an optional part of the intervention to the first group of subjects. ${ }^{19}$ However, due to high inter-individual variation and nonadherence with intake of supplements, we did not provide nutritional supplements to the second group of subjects. Similarly, the first group was 
recommended to use sub-motor intensity electrical stimulation during activities of daily living; however, only three subjects used this option. Thus, to simplify the intervention, we did not recommend this option to the second group. Overweight and obese subjects in the first group experienced $>10 \%$ weight loss from baseline to 12 months. ${ }^{19}$ Thus, we started to monitor subjects' weights at each visit and subjects were instructed to increase intake of animal protein and coconut oil to prevent excessive weight loss. If $>10 \%$ weight loss from baseline to 6 months was recorded, subjects' primary-care doctors were informed.

\section{Outcome measures}

\section{Gait and balance assessments}

A physical therapist (B. Bisht) conducted tests of gait (TUG and T25FW tests) and balance (Berg Balance Scale). TUG test was considered primary outcome measure and T25FW and Berg Balance Scale scores were considered secondary outcome measures. The following instructions were given to the subjects for the TUG: "On the word go, stand up from the chair, walk up to 10 feet mark, turn around, walk back to the chair and sit down. Walk with your comfortable speed". Timing for the test began as subjects leaned forward to stand up and was stopped as their pelvis touched the seat of the chair. For T25FW, subjects were instructed to "walk as fast as possible but safely" to a distance of 25 feet. For this test, we started timing as subject's leading foot crossed the start line and finished timing when both feet crossed the finish line. Percentage change in time to complete TUG at post-intervention visits compared with baseline was the primary outcome measure. TUG tests were also video recorded for off-line gait analyses using two cameras (for side view, SONY HDR-CX150 Sony Corporation, Tokyo, Japan and for frontal view Cisco Flip UltraHD 3 [U32120], Cisco Systems Inc., San Jose, CA, USA). Duration of the following parts of TUG test were assessed manually from videos by a trained research assistant: 1) stand from seated position, 2) walk to 10 feet mark, 3) turn around, 4) walk back to chair from 10 feet mark, and 5) sit from standing position.

\section{Spatio-temporal gait parameter analysis}

Two-dimensional video gait analyses were performed using SIMI movement analysis package (SIMI Reality Motion System, Unterschleissheim, Germany) to assess the following gait parameters (secondary outcome measures) from TUG videos: longer and shorter step lengths (chosen based on subject's step lengths at baseline), step and stride duration and double support time. Two-dimensional video gait analysis is a reliable method and assessment of spatio-temporal gait parameters using this method are shown to correlate highly with those assessed with instrumented walkways such as the GAITRite mat (CIR Systems Inc., Franklin NJ, USA) (r $\geq 0.94) .{ }^{21}$ Calibration of the motion analysis system was achieved by inputting the coordinates of a rectangular frame of reference just behind the subject. Subjects' feet were manually digitized at heel strike and toe off phase of gait cycle to calculate gait parameters. For subjects who did not demonstrate clear heel strike and toe off during walking and dragged their foot, heel was digitized at the time when subject stopped moving the foot forward. For these subjects, the toes were digitized when the foot began to move forward for the next step. Prior to TUG of each subject, a video of a rod with black tape markers at known distances, placed horizontally on the floor, was recorded and used to assess validity of SIMI motion system measures of distance between the tape markers.

\section{Reliability of gait and balance tests}

Gait (TUG and T25FW) and balance (Berg Balance Scale) tests were video recorded at the time of assessment and analyzed from videos by a research assistant who was blinded to subject's visits. The research assistant's (second rater) analyses were then used to assess reliability of the physical therapist's (first rater) assessments. Although, video recordings of TUG and T25FW were performed from the initiation of the study trial, video recordings of Berg Balance Scale for all subjects were started at enrollment of the ninth subject of the study (subject 14).

\section{Perceived fatigue}

Perceived fatigue was measured with a self-reported questionnaire - fatigue severity scale (FSS). FSS is a 9-item questionnaire in which subjects are asked to rate how fatigue affects their activities of daily life. FSS is a reliable and sensitive measure, and has demonstrated internal consistency and discriminative properties in pwMS. ${ }^{22}$

\section{Statistical analysis}

In this intent to treat analysis all available data were used. There were 2 subjects with missing data due to inability to perform walking task in some testing sessions after baseline and 1 subject who was withdrawn due to cognitive decline at 6 months. For these subjects, missing data for TUG, time to stand up, time to turn, time to sit, and double support time during TUG test were assigned a value of longest observed time in the group $+1 \mathrm{~s}$. Time to walk to 10 feet mark and time to walk back to chair from the 10 feet mark were converted into average speed $(\mathrm{cm} / \mathrm{s})$, and subjects with missing values 
Table I Detectable mean change in outcome measures for sample size of $n=20$ at 0.05 significance level with 0.80 power

\begin{tabular}{lll}
\hline Variable & $\begin{array}{l}\text { CV }(\%) \\
\text { or SD of } \\
\text { change }\end{array}$ & $\begin{array}{l}\text { Detectable } \\
\text { change }\end{array}$ \\
\hline TUG & $46 \%$ & $30 \%$ \\
First I0 feet walk speed during TUG $(\mathrm{cm} / \mathrm{s})$ & 15.2 & 11.6 \\
TUG-short step length $(\mathrm{cm})$ & 8.2 & 6.3 \\
TUG-long step length $(\mathrm{cm})$ & 9.2 & 7.1 \\
I/TUG step duration $(\mathrm{step} / \mathrm{s})$ & 0.25 & 0.19 \\
T25FW speed $(\mathrm{cm} / \mathrm{s})$ & 13.4 & 10.3 \\
\hline
\end{tabular}

Abbreviations: $\mathrm{CV}$, coefficient of variation; $\mathrm{SD}$, standard deviation; TUG, timed up and go; T25FW, timed 25-foot walk.

were assigned a speed of $0 \mathrm{~cm} / \mathrm{s}$. Similarly, missing data for $25 \mathrm{FW}$ speed was replaced with $0 \mathrm{~cm} / \mathrm{s}$. Missing data for step lengths (longer and shorter), Berg Balance Scale scores, adherence with study diet and NMES and exercise program were assigned a value of zero. Reciprocal transformation was used for step and stride duration to normalize data distribution and for missing values, a value of zero was used. For each of the outcome measures, the detectable mean change for a sample size of $n=20$ at the 0.05 significance with 0.80 power was calculated (Table 1).

Linear mixed model analysis for repeated measures was used to test for change over time in TUG test time and its parts and spatio-temporal gait parameters, and T25FW speed with Dunnett's test used for pairwise comparisons to assess change from baseline at different time points during the study. For variables that were not normally distributed, a natural log or reciprocal transformation was applied to the data to normalize the data distribution. For the transformed variables, mean in the original scale was computed by back-transformation, with the corresponding standard error (SE) calculated using the delta method. Friedman's test was used to test for change in adherence with study diet, adherence with NMES-exercise program, and Berg Balance Scale scores. Previously, we reported significant decrease in fatigue of study participants within 3 months ${ }^{20}$ and thus to investigate if change in fatigue within 3 months had any effect on walking performance during study period, we performed Pearson correlations between changes in FSS scores from baseline to 3 months and changes in walking performance during TUG and T25FW tests from baseline to 12 months. Inter-rater reliability of TUG, T25FW, and Berg Balance Scale scores were assessed by intraclass correlation between the values recorded by the first rater during the test sessions and values obtained by another rater from video recordings of these tests on 20 randomly chosen videos, 4 at each visit.
We found that $50 \%$ of the subjects $(n=10)$ showed decrease in TUG test time (improved performance) on at least 3 of the 4 visits after the initiation of the intervention, whereas remaining subjects did not show improvement on at least 3 of those 4 visits. We considered subjects with improved performance in TUG test as responders (TUG-Res) and the remaining subjects were considered as nonresponders (TUG-NRes). To assess how these two groups had responded for other outcome variables, the mean change (with 95\% confidence interval [CI]) at 6, 9, and 12 months for other clinical outcomes such as TUG parts, spatio-temporal gait parameters, T25FW speed, and Berg Balance Scale scores were calculated. In addition, to investigate subject characteristics and baseline function that may differentiate between TUG-Res and TUG-NRes, baseline characteristics (age, sex, disease-modifying drug use, MS duration, EDSS scores, time to complete TUG and TUG parts, spatio-temporal gait parameters, $\mathrm{T} 25 \mathrm{FW}$ speed, and Berg Balance Scale scores) as well as adherence to the intervention components were compared using 2-sample $t$-test (for normally distributed data) and Wilcoxon rank-sum test (for non-normally distributed data). All analyses were performed using SAS software, Version 9.4 (SAS Institute Inc., Cary, NC, USA)

\section{Results \\ Participants}

Twenty six subjects were enrolled in the run-in phase, of which 21 met eligibility criteria for 12-month main study. One subject (subject 10) withdrew from the study within 3 months for unknown reasons (subject did not show up for 3-month visit and did not return phone calls from study team). We did not include this subject's data as only baseline data were available. Thus, data of 20 subjects who continued beyond 3 months in the study were included in the analysis. One subject (subject 8) was withdrawn at 6 months due to clinically significant cognitive decline. Mean age of the 20 subjects was 51.7 (standard deviation $[\mathrm{SD}]=6.4$ ) years, average disease duration was 14.7 $(\mathrm{SD}=8.7)$ years and average EDSS scores were 6.2 $(\mathrm{SD}=1)$. Detailed demographics and baseline clinical characteristics of the subjects were reported previously. ${ }^{20}$ Subject's screening, enrollment, and follow-up during the study are shown in Figure 1 and were also reported previously. ${ }^{20}$

\section{Intervention}

A detailed description of subjects' adherence and dosage of each components of the intervention (study diet, NMES, exercises, meditation, and massage) was provided previously. ${ }^{20}$ In summary, subjects showed a median dietary adherence ranging from $99.5 \%$ to $100 \%$ and 


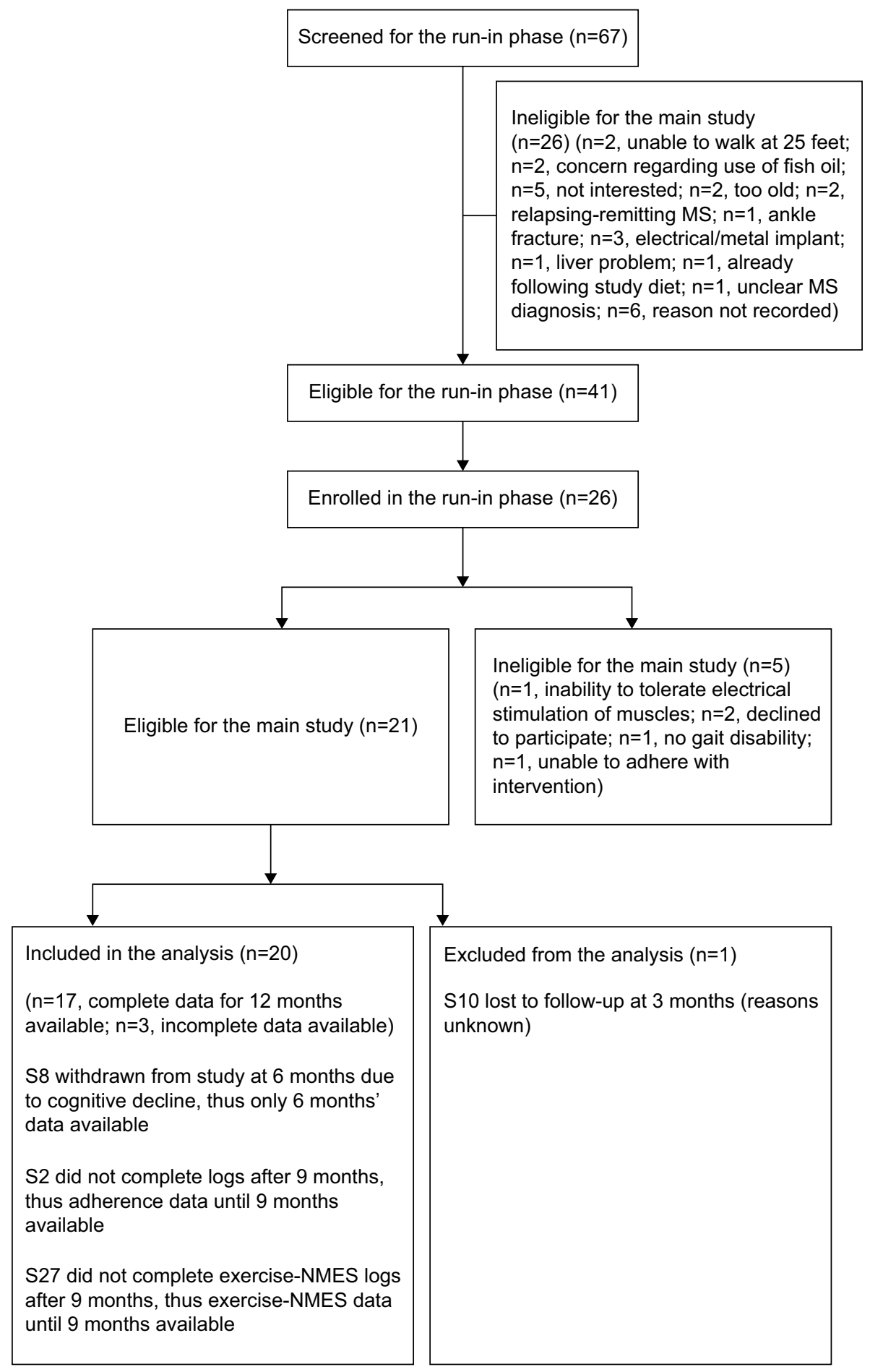

Figure I Flowchart of the screening, enrollment, and follow-up of participants in the study.

Source: Copyright @2014. Dove Medical Press. Reproduced from Bisht B, Darling WG, Shivapour ET, et al. Multimodal intervention improves fatigue and quality of life of subjects with progressive multiple sclerosis: a pilot study. Degenerat Neurol Neuromuscul Dis. 2015;2015(5): 19-35. ${ }^{20}$

Abbreviations: S, subject; NMES, neuromuscular electrical stimulation; MS, multiple sclerosis.

NMES-exercise adherence ranging from $81.4 \%$ to $91.3 \%$ during 12 months. No significant changes in dietary and NMES-exercise adherence were observed during 12 months $(p>0.32)$. A detailed description of side effects was provided previously. ${ }^{20}$ No serious side effects were reported during the study.

\section{Gait and balance assessments}

\section{Reliability}

Intraclass correlation between the measurements of the first and second rater showed high reliability (absolute agreement) of the measurement of TUG (intraclass correlation coefficient [ICC]: 1.000; 95\% CI: 0.999-1), T25FW (ICC: 1.000; 95\% 
CI: 0.999-1) and Berg Balance Scale scores (ICC: 0.992; 95\% CI: 0.969-0.997).

\section{TUG, T25FW, and Berg Balance test}

For the entire cohort, no significant changes in time to complete TUG ( $p=0.600$, Figure 2$)$, spatio-temporal gait parameters (all $p>0.13$ ) and T25FW speed ( $p=0.732$, Figure3) were observed. Mean (SE) TUG time was $37.03 \mathrm{~s}(8.89)$ at baseline and $37.25 \mathrm{~s}(8.94)$ at 12 months. Mean (SE) T25FW speed for the cohort was $43.7 \mathrm{~cm} / \mathrm{s}(8.7)$ at baseline and 50.8 $\mathrm{cm} / \mathrm{s}(9.8)$ at 12 months. Analysis of parts of TUG suggested an increase from baseline in speed of walking toward 10 feet mark during the study ( $p=0.082$ ). Pairwise comparison using Dunnett's test showed significant increase in speed at 6 months compared with baseline ( 40.54 vs $48.34 \mathrm{~cm} / \mathrm{s}$,

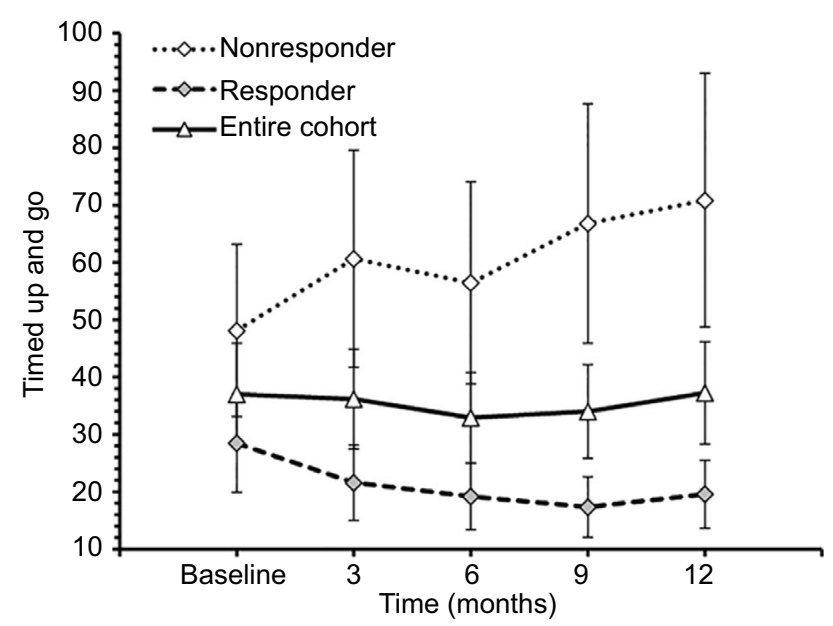

Figure 2 Time to complete timed up and go test at baseline, 3, 6, 9, and 12 months of the entire cohort $(n=20)$, responders $(n=10)$ and nonresponders $(n=10)$.

Note: Values are mean \pm standard error.

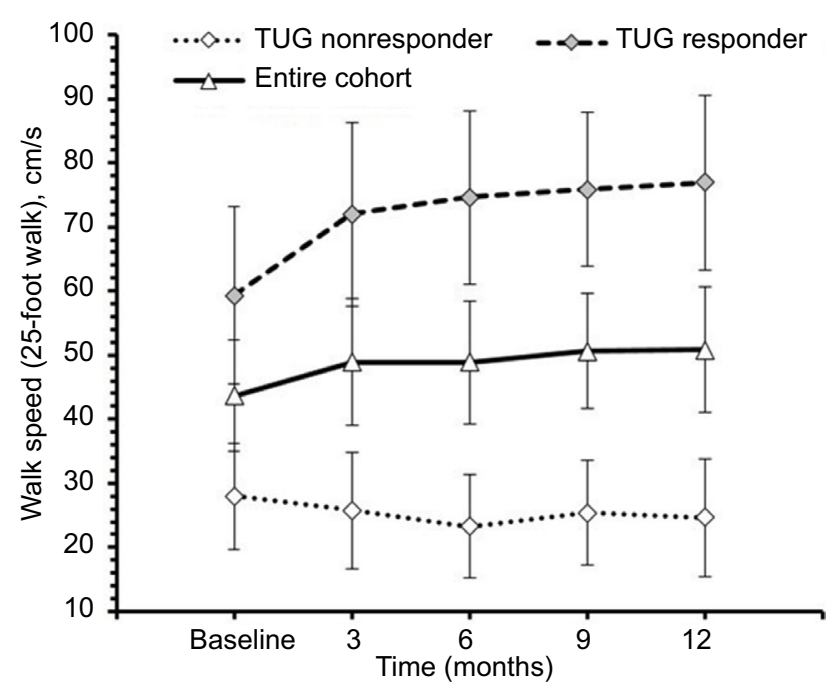

Figure 3 Walking speed during 25-foot walk test at baseline, 3, 6, 9, and I 2 months of the entire cohort $(n=20)$, responders $(n=10)$ and nonresponders $(n=10)$.

Note: Values are mean \pm standard error.

Abbreviation: TUG, timed up and go. adjusted $p=0.041$, Figure 4); however, change in speed at 9 and 12 months from baseline were not statistically significant. The mean change $(95 \% \mathrm{CI})$ at 6,9 , and 12 months for these outcome measures are presented in Table 2.

The effect of disease-modifying drugs on TUG and T25FW speed was assessed by including medication in the linear mixed model. This showed no significant effects of medication on mean change in TUG $(p=0.78)$, walking toward 10 feet mark during TUG $(p=0.85)$, and T25FW speed $(p=0.49)$ at 6,9 , and 12 months.

For the entire cohort, Friedman's test showed no significant change in Berg Balance Scale scores over 12 months ( $p=0.259$, Figure 5 ). Median scores (interquartile range) were $38(5.5-44.5)$ at baseline and changed to $37.5(6.75-47)$ at 12 months. When assessed for change in number of tasks subjects could perform from baseline to 12 months, 7 subjects showed increase, 6 subjects showed decrease, and the remainder did not show any change.

\section{Responders vs nonresponders}

There was high interindividual variability in subjects' response to the intervention. Upon detailed analysis, a dichotomous response to the intervention was found among subjects. From the entire cohort, 10 subjects ( 8 secondary progressive, 2 primary progressive) showed decrease in time to complete TUG from baseline on at least 3 of 4 timepoints post-intervention (considered as TUG-Res), whereas other 10 did not show improved TUG on at least 3 of 4 post-intervention tests (considered as TUG-NRes). It was

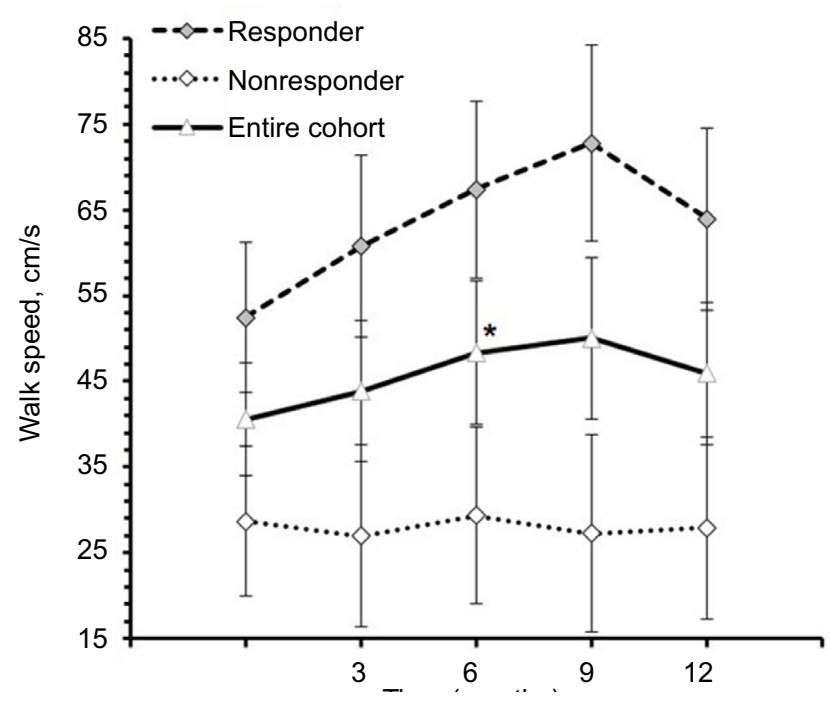

Figure 4 Speed to walk toward 10 feet mark during timed up and go test at baseline, 3, 6, 9, and 12 months of the entire cohort $(n=20)$, responders $(n=10)$ and nonresponders $(n=10)$.

Notes: Values are mean \pm standard error. ${ }^{*} p=0.04 \mathrm{I}$, significant difference from baseline for entire cohort. 
Table 2 Mean change $(95 \% \mathrm{Cl})$ at 6,9 , and 12 months (all subjects)

\begin{tabular}{llll}
\hline Variable & $\mathbf{6}$ months & $\mathbf{9}$ months & I2 months \\
\hline TUG & $-11 \%(-32 \%, 17 \%)$ & $-8 \%(-34 \%, 28 \%)$ & $-0.6 \%(-31 \%, 47 \%)$ \\
First 10 feet walk speed during TUG $(\mathrm{cm} / \mathrm{s})$ & $7.9(0.3,15.2)$ & $9.4(-1.8,21.0)$ & $5.5(-4.9,15.5)$ \\
TUG-short step length $(\mathrm{cm})$ & $2.8(-1.7,7.4)$ & $1.5(-3.0,6.1)$ & $1.7(-2.9,6.2)$ \\
TUG-long step length $(\mathrm{cm})$ & $1.4(-3.4,6.2)$ & $0.4(-5.1,5.9)$ & $-0.8(-5.5,3.9)$ \\
I/TUG step duration $($ step/s) & $0.07(-0.07,0.21)$ & $0.01(-0.12,0.15)$ & $0.02(-0.12,0.16)$ \\
T25FW speed $(\mathrm{cm} / \mathrm{s})$ & $5.3(-2.7,13.2)$ & $7.0(-2.6,16.5)$ & $7.1(-3.9,18.1)$ \\
\hline
\end{tabular}

Abbreviations: $\mathrm{Cl}$, confidence interval; TUG, timed up and go; T25FW, timed 25-foot walk.

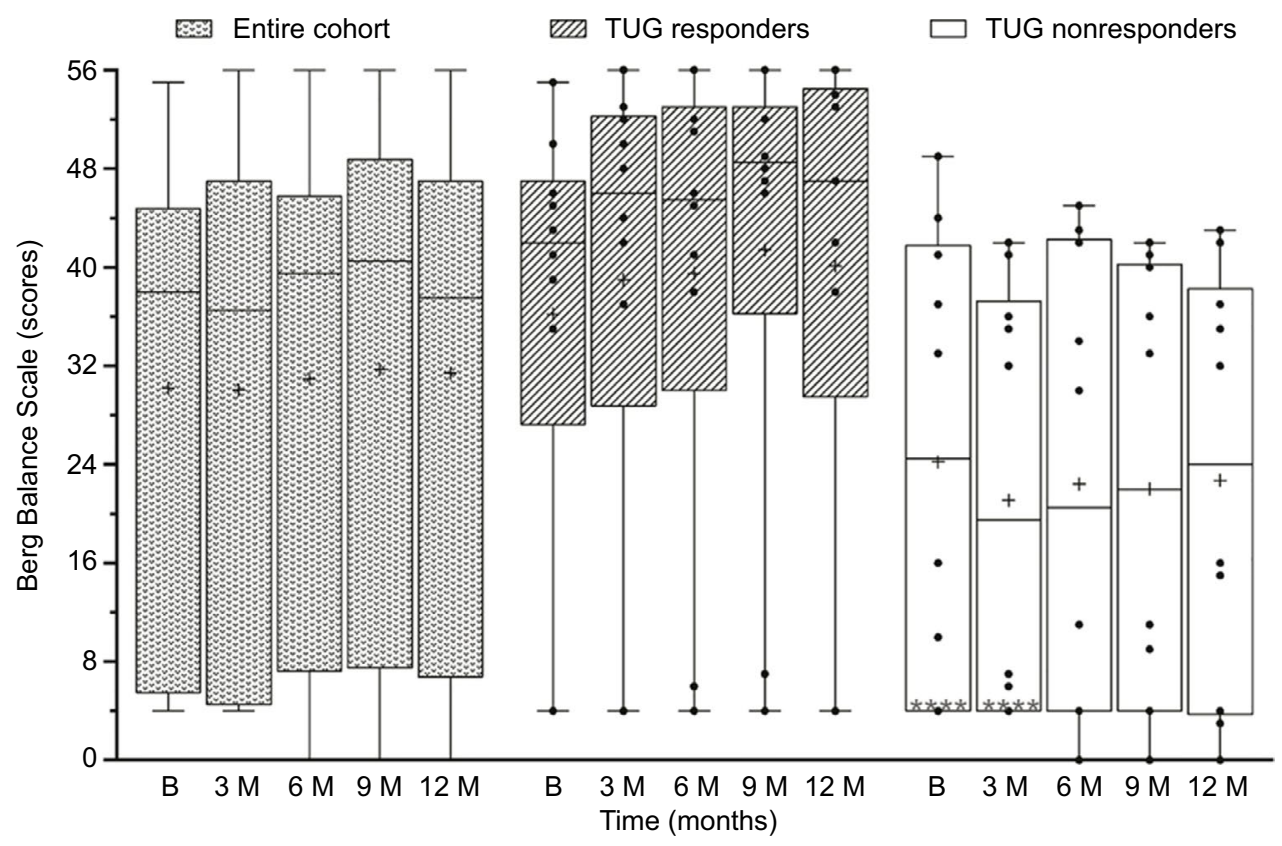

Figure 5 Berg Balance Scale scores of the entire cohort $(n=20)$, and responders $(n=10)$ and nonresponders $(n=10)$ to TUG test at baseline (B), 3, 6, 9, and I2 months. Notes: Upper and lower borders of the boxes represent 25th and 75th percentiles, respectively. The line within the boxes represents median and + sign represents mean. The whisker error bars show minimum and maximum values. **** represent lower quartile values overlapping with minimum values. Each black symbol represents individual subject's score.

Abbreviation: TUG, timed up and go.

expected there may be some TUG-NRes because all subjects had progressive MS and would typically decline in TUG performance over 12 months. ${ }^{18,23,24}$ The mean changes (with $95 \% \mathrm{CI})$ in gait and balance outcomes were characterized for TUG-Res and TUG-NRes at 6, 9, and 12 months (Table 3).

Mean (SE) TUG time of TUG-Res was $28.51 \mathrm{~s}(8.63)$ at baseline, which decreased to $19.59 \mathrm{~s}$ (5.93) at 12 months (Figure 2). Some example videos are presented to show improved gait and balance over 12 months of some TUG-Res (Video S1: 00:00:01:04 - 00:02:20:12). Subject 3 was at baseline 1 of the most disabled subjects in the study. During her baseline TUG test (Video S1: 00:00:01:04), this subject showed poor balance, needed assistance to stand from seated position, had difficulty clearing her feet while walking and research team had to follow the subject with a chair due to risk of fall. However, despite severe disability at baseline, this subject showed visible improvements in her gait and balance at 9-month visit (Video S1: 00:00:51:08). The video shows that the subject was able to stand up from seated position by just pulling on the hand rail, had better foot clearance, and showed improved balance. Subject 11 showed dramatic improvement in her gait and balance within 3 months of following the intervention. At baseline, subject 11 was using a cane to walk (Video S1: 00:01:07:18). However, at 3 months, this subject experienced great improvement in her gait and balance, such that she did not need the cane anymore and was able to walk normally (Video S1: 00:01:16:00). It should be noted that the subject had a such high level of confidence in the swing phase of her gait and ankle dorsiflexion that she wore sandals without a behind-theheel ankle strap. Furthermore, this subject was able to jog at 6 months post-intervention (Video S1: 00:01:22:10). Subject 14 needed two walking poles to maintain balance during baseline TUG (Video S1: 00:01:27:10), whereas at 9 months, was able to walk independently and showed clear improvement in his balance (Video S1: 00:01:45:00). Subject 17, who had primary progressive MS (PPMS), also showed improved gait and bal- 
Table 3 Mean change $(95 \% \mathrm{Cl})$ at 6, 9, and 12 months for responder and nonresponder

\begin{tabular}{|c|c|c|c|}
\hline Responder & 6 months & 9 months & 12 months \\
\hline TUG (\%) & $-33(-48,-13)$ & $-39(-55,-17)$ & $-31(-52,-2)$ \\
\hline First 10 feet walk speed during TUG $(\mathrm{cm} / \mathrm{s})$ & $14.9(5.2,24.7)$ & $20.4(5.8,34.7)$ & II.6 (-3.0, 25.9) \\
\hline TUG-short step length (cm) & $6.8(0.9,12.8)$ & $7.7(1.8,13.6)$ & $6.9(0.9,12.8)$ \\
\hline TUG-long step length $(\mathrm{cm})$ & $4.9(-1.6,11.3)$ & $5.4(-1.6,12.5)$ & $3.6(-2.3,9.6)$ \\
\hline I/TUG step duration (step/s) & $0.16(0.08,0.27)$ & $0.20(0.08,0.31)$ & $0.20(0.08,0.32)$ \\
\hline T25FW speed $(\mathrm{cm} / \mathrm{s})$ & $15.3(2.6,27.9)$ & $16.6(1.2,32.0)$ & $17.6(0.01,35.2)$ \\
\hline \multicolumn{4}{|l|}{ Nonresponder } \\
\hline TUG (\%) & $17(-25,84)$ & $39(-19,138)$ & $47(-20,170)$ \\
\hline First I0 feet walk speed during TUG $(\mathrm{cm} / \mathrm{s})$ & $0.6(-9.1,10.4)$ & $-1.5(-15.8,13.1)$ & $-0.6(-15.2,13.7)$ \\
\hline TUG-short step length (cm) & $-1.2(-7.1,4.7)$ & $-4.6(-10.5,1.3)$ & $-3.5(-9.4,2.4)$ \\
\hline TUG-long step length $(\mathrm{cm})$ & $-2.0(-8.5,4.4)$ & $-4.6(-11.6,2.4)$ & $-5.3(-11.2,0.7)$ \\
\hline I/TUG step duration (step/s) & $-0.02(-0.25,0.21)$ & $-0.17(-0.40,0.06)$ & $-0.16(-0.39,0.06)$ \\
\hline T25FW speed $(\mathrm{cm} / \mathrm{s})$ & $-4.7(-12.8,3.3)$ & $-2.6(-12.4,7.2)$ & $-3.4(-14.6,7.9)$ \\
\hline
\end{tabular}

Abbreviations: $\mathrm{Cl}$, confidence interval; TUG, timed up and go; T25FW, timed 25 -foot walk.

ance. She needed to use 2 canes to maintain balance during TUG at baseline (Video S1: 00:01:54:19) but at 3 months, was using only 1 cane to ambulate at home and outside and was even able to ambulate independently without risk of fall (Video S1: 00:02:02:00 and 00:02:10:19). We would like to point out that for both subjects 14 and 17, post-intervention gait analysis was performed on videos with use of assistive devices and videos showing independent ambulation are presented to visually demonstrate substantial gait improvements in these subjects.

TUG-NRes showed increase in TUG time (mean, SE) from $48.11 \mathrm{~s}(15.02)$ at baseline to $70.85 \mathrm{~s}(22.13)$ at 12 months (Figure 2). Some example videos are presented to show decline in gait and balance over 12 months in these subjects (Video S1: 00:02:21:00 - 00:03:36:00). For example, subject 9 experienced rigidity in her legs and walked slowly at baseline using a cane (Video S1: 00:02:21:00). At 12 months (Video S1: 00:02:35:10), no change in her balance was observed from baseline (Berg Balance Scale scores, 41 vs 42); however, her gait speed decreased (TUG time, 29.2 vs $33.2 \mathrm{~s}$ ). Subject 15 had difficulty clearing her feet off the ground, suggesting decreased muscle strength in both legs at 12 months (Video S1: 00:03:02:04 - note absence of dorsiflexion at initial contact, suggesting weak dorsiflexors in both legs, and rising up on toes of right foot at the end of stance phase, suggesting weak hip/knee flexors of left leg) compared with baseline (Video S1: 00:02:51:09) and resulting in slower gait speed. Subject 22 showed increased forward trunk lean during stance phase of each leg from baseline to 12 months, suggesting quadriceps weakness (Video S1: 00:03:14:10 vs 00:03:24:05). Also note reduced knee flexion and ankle dorsiflexion of the left leg, suggesting weak hamstrings and dorsiflexors at 12 months compared with baseline.

Among TUG-Res, 6 of 10 subjects showed clinically significant improvement in TUG test $(>24 \%$ decrease in time), ${ }^{25}$ whereas among TUG-NRes, 5 of 10 subjects showed clinically significant deterioration in TUG test $(>31 \%$ increase in time $)^{25}$ at 12 months in relation to baseline. For TUG-Res, mean (SE) speed during walking toward 10 feet mark increased from $52.45 \mathrm{~cm} / \mathrm{s}(8.74)$ at baseline to 63.92 $\mathrm{cm} / \mathrm{s}(10.58)$ at 12 months (Figure 4). For TUG-NRes, speed (mean, SE) decreased from $28.63 \mathrm{~cm} / \mathrm{s}(8.74)$ at baseline to $27.88 \mathrm{~cm} / \mathrm{s}(10.58)$ at 12 months (Figure 4). No significant changes were observed in speed to stand up from chair, to sit down, to turn and walk 10 feet back to chair. TUG-Res showed increases in longer and shorter step lengths averaging $5-7 \mathrm{~cm}$ as well as small decreases in step duration over 12 months (Figure 6), whereas TUG-NRes showed $\sim 5 \mathrm{~cm}$ decreases in longer and shorter step lengths and clear increases in step duration from baseline to 12 months (Figure 6).

TUG-Res showed a $30 \%$ increase in mean T25FW speed over 12 months $(59.3 \mathrm{~cm} / \mathrm{s}$ [13.8] to $76.9 \mathrm{~cm} / \mathrm{s}$ [13.6], Figure 3), whereas TUG-NRes showed a $12 \%$ decrease in mean T25FW speed $(28.0 \mathrm{~cm} / \mathrm{s}$ [8.3] to $24.6 \mathrm{~cm} / \mathrm{s}$ [9.2], Figure 3). Among TUG-Res, 7 of 10 subjects showed clinically significant increase in T25FW speed $(>20 \%)^{26}$ from baseline to 12 months, whereas 4 of 10 TUG-NRes showed clinically significant decrease in gait speed over 12 months.

Median Berg Balance Scale scores (interquartile range) of TUG-Res increased from 42 (27.3-47) at baseline to 47 (29.5-54.5) at 12 months, whereas TUG-NRes did not show much change over 12 months, 24.5 (4-41.8) at baseline and 24 (3.8-38.3) at 12 months (Figure 5). Among TUG-Res, 2 subjects showed increase in scores greater than minimal detectable change ( 7 points) ${ }^{27}$ at 12 months, whereas among TUG-NRes, 3 subjects showed minimal detectable decrease and 2 subjects showed minimal detectable increase in scores, suggesting highly variable response to this test. 
A

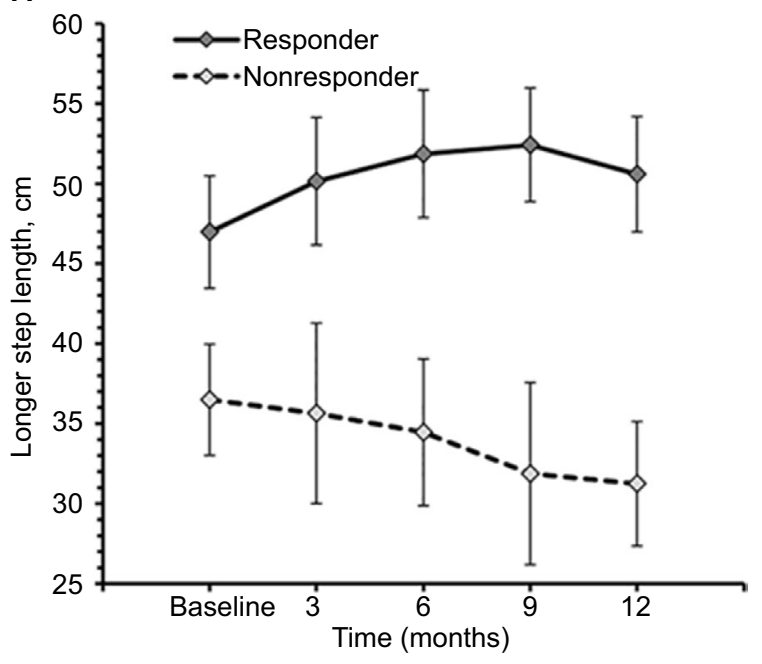

B

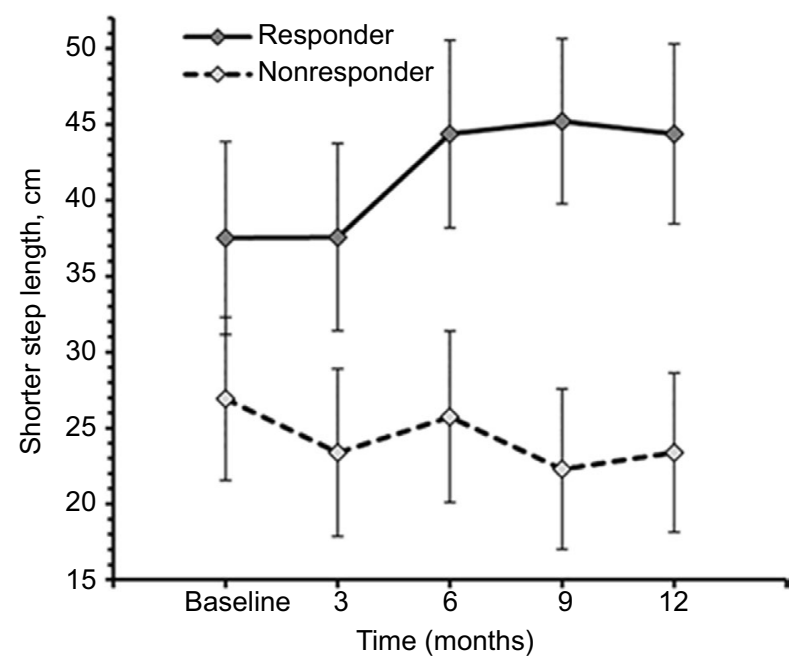

C

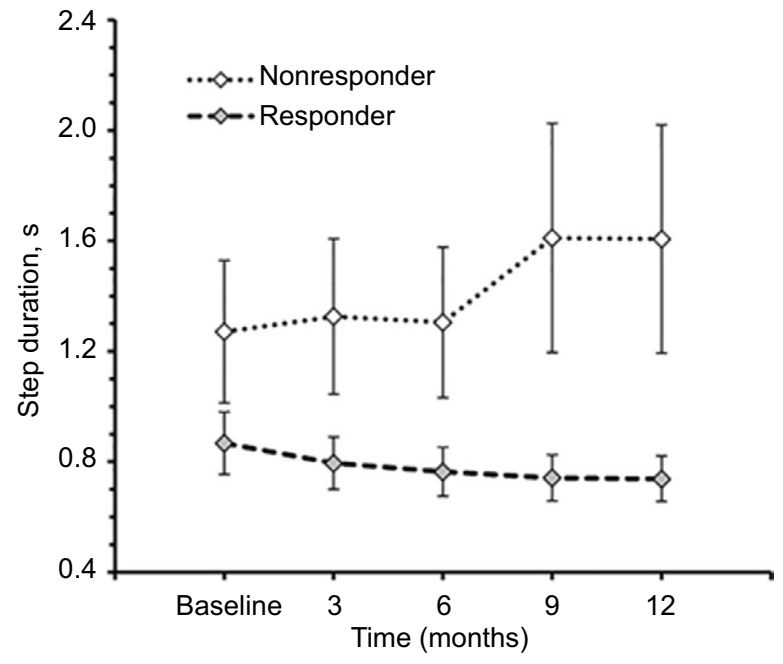

Figure 6 Spatio temporal gait parameters during timed up and go test at baseline, 3, 6, 9, and 12 months of responders ( $n=10)$ and nonresponders ( $n=10$ ). Notes: (A) longer step length, (B) shorter step length, and (C) step duration. Values are mean \pm standard error.

Comparison of the baseline characteristics of TUG-Res and TUG-NRes (Table 4) showed that TUG-Res had greater TUG longer step length (mean: 46.97 vs $36.5 \mathrm{~cm}, p=0.048$ ) and shorter stride duration ( 1.64 vs $2.84 \mathrm{~s}, p=0.041$ ) compared with TUG-NRes. The data also suggested differences in parts of the baseline TUG test with TUG-Res taking less time than TUG-NRes: time to sit ( 3.3 vs $7.8 \mathrm{~s}, p=0.075$ ), time to turn ( 3.3 vs $9.1 \mathrm{~s}, p=0.089$ ), time to walk toward 10 feet mark (5.2 vs $14.7 \mathrm{~s}, p=0.076)$, and time to walk 10 feet back to chair (5.2 vs $13.8 \mathrm{~s}, p=0.059$ ). Also, among TUG-Res, only 2 of 10 subjects were using rolling walker, whereas 6 of 10 TUG-NRes were using rolling walker or rollator at baseline. Thus, differences in baseline function may be associated with responses to the intervention.

Friedman test showed no significant change in dietary adherence during the follow-up period within both the responder $(p=0.244)$ and nonresponder $(p=0.509)$ groups.
Comparison at each follow-up using Wilcoxon rank-sum test showed no significant difference in adherence to diet between responder and nonresponder $(p>0.17)$. Similar results were found for adherence to NMES-exercise program, with no significant change in adherence with NMESexercise program during the follow-up period within both the responder $(p=0.344)$ and nonresponder $(p>0.99)$ groups, and no significant difference in adherence between responder and nonresponder groups $(p>0.99)$. Median compliance at 12 months was $98 \%$ for diet and $83 \%$ for exercise in TUGRes, and $100 \%$ for diet and $81 \%$ for exercise in TUG-NRes.

\section{Association between change in FSS score at 3 months and change in walking performance at 12 months}

Changes in FSS scores at 3 months in relation to baseline were inversely correlated with changes in $\mathrm{T} 25 \mathrm{FW}$ speed over 
Table 4 Baseline variables - responder vs nonresponder

\begin{tabular}{|c|c|c|c|}
\hline Variable & Responder $(n=10)$ & Nonresponder $(n=10)$ & $p$-value \\
\hline Age (years), mean (SD) & $52.0(7.7)$ & $51.5(5.3)$ & 0.878 \\
\hline Number of females (\% of group) & $7(70 \%)$ & $8(80 \%)$ & 1.0 \\
\hline MS duration (years) & I2.I (6.4) & $17.2(10.3)$ & 0.200 \\
\hline EDSS (score), median (IQR) & $6.25(6.0-6.5)$ & $6.50(6.0-7.0)$ & 0.236 \\
\hline DMD use (\% of group) & $4(40 \%)$ & $3(30 \%)$ & 1.0 \\
\hline FSS (score) & $5.85(5.17)$ & $5.13(4.10)$ & 0.205 \\
\hline TUG time - total (s) & $22.2(14.9-26.7)$ & $53.2(26.1-81.8)$ & 0.186 \\
\hline TUG time - stand (s) & $2.5(1.2-3.1)$ & $3.2(1.9-5.1)$ & 0.449 \\
\hline TUG time - sit $(s)$ & $3.3(3.2-4.5)$ & $7.8(5.4-15.7)$ & $0.075^{*}$ \\
\hline TUG time - turn (s) & $3.3(2.8-4.3)$ & $9.1(5.3-15.0)$ & $0.089 *$ \\
\hline TUG time - first 10 feet (s) & $5.2(4.3-8.9)$ & $14.7(6.6-23.8)$ & $0.076 *$ \\
\hline TUG time - second 10 feet $(\mathrm{s})$ & $5.2(3.2-6.9)$ & $13.8(8.1-23.1)$ & $0.059 *$ \\
\hline TUG gait - long step (cm) & $46.97(11.13)$ & $36.50(11.01)$ & $0.048^{* *}$ \\
\hline TUG gait - short step $(\mathrm{cm})$ & $46.09(34.13-50.01)$ & $27.10(22.40-38.26)$ & 0.112 \\
\hline TUG gait - step duration (s) & $0.84(0.67-1.10)$ & $\mathrm{I} .40(0.9 \mathrm{I}-2.86)$ & $0.069 *$ \\
\hline TUG gait - stride duration (s) & $1.64(1.34-1.84)$ & $2.84(2.17-5.76)$ & $0.04 I^{* *}$ \\
\hline TUG gait - double support duration (s) & $0.23(0.11-0.38)$ & $0.77(0.33-\mid .3 I)$ & 0.121 \\
\hline T25FW speed $(\mathrm{cm} / \mathrm{s})$ & | $3.1(9.7-25.8)$ & $41.0(16.5-98.5)$ & 0.104 \\
\hline Berg balance test (score) & $42.0(35.0-46.0)$ & $24.5(4.0-41.0)$ & 0.148 \\
\hline
\end{tabular}

Notes: ${ }^{*} p<0.1 ;{ }^{* *} p<0.05$. Data presented as mean (SD), $\mathrm{n}(\%)$ or median (IQR).

Abbreviations: MS, multiple sclerosis; EDSS, expanded disability status scale; IQR, interquartile range; DMD, disease modifying drug; FSS, fatigue severity scale; SD, standard deviation; TUG, timed up and go; T25FW, timed 25-foot walk.

12 months in the entire cohort $(\mathrm{r}=-0.69,95 \% \mathrm{CI}:-0.87$, $-0.36, p=0.0004$; Figure 7 ), suggesting that improvements in fast walking speed over 12 months were related to early improvements in perceived fatigue. This correlation remained strong when controlled for baseline $\mathrm{T} 25 \mathrm{FW}$ speed $(\mathrm{r}=-0.73$, 95\% CI: $-0.89,-0.41, p=0.0002$; Figure 7). In contrast, change in time to complete TUG over 12 months showed no significant correlation with change in fatigue scores over 3 months ( $\mathrm{r}=0.39,95 \% \mathrm{CI}:-0.06,0.71, p=0.083)$ and similarly, no significant correlation were observed when controlled for baseline TUG time ( $\mathrm{r}=0.22,95 \% \mathrm{CI}:-0.26,0.61, p=0.366)$ suggesting that subject's ability to perform TUG test, which included standing up, turning around, sitting down, and walking at comfortable speed, is unrelated to change in FSS scores over 3 months.

\section{Discussion}

We did not find significant changes in gait and balance outcomes in our study cohort following a multimodal intervention for 12 months. It is noteworthy that these nonsignificant results were mainly because of high variability in baseline characteristics of study subjects that probably led to a variable response to the intervention. Fifty percent $(n=10)$ of subjects responded positively, whereas the other $50 \%$ either did not show any change or showed deterioration in their gait and balance functions. Sub-group analyses showed that subjects who responded favorably to the intervention had comparatively less gait impairment at baseline than the subjects who did not respond well to the intervention. Importantly, for the entire cohort, we found that greater decrease in perceived fatigue over the initial 3 months of the intervention was associated with improved performance during fast walking task at 12 months, suggesting that subjects who respond better early to this intervention in terms of perceived fatigue may respond well in terms of gait impairments as well. Thus, our pilot study provides information that may help select pwMS who will respond well to this multimodal intervention.

Slowing of gait negatively impacts activities of daily living, participation in the community, and quality of life for pwMS. ${ }^{3,28,29}$ Recent studies have shown that there are reductions in gait speed over 12 months in most individuals with progressive MS. ${ }^{18,23,24}$ For example, Vermersch et al reported average relative increases of $26 \%(\mathrm{SD}=55)$ and $5 \%$ $(\mathrm{SD}=26)$ in time to complete $\mathrm{T} 25 \mathrm{FW}$ test over 12 months, respectively, in a placebo group of 6 subjects and a treatment group (receiving masitinib) of 24 subjects with primary and secondary progressive MS. ${ }^{18}$ Another study assessing the effects of lamotrigine in subjects with SPMS reported 12\% increase in $\mathrm{T} 25 \mathrm{FW}$ time in placebo group over a period of 12 months. ${ }^{23}$ Thus, it is important to identify interventions to prevent or, ideally, reverse established gait impairment in progressive MS. Although 50\% ( $\mathrm{n}=10$, TUG-NRes) of subjects showed trends toward deterioration in walking performance and balance from baseline to 12 months, as would be expected of most individuals with progressive MS, the other $50 \%$ (TUG-Res) showed trends toward improved walking ability 


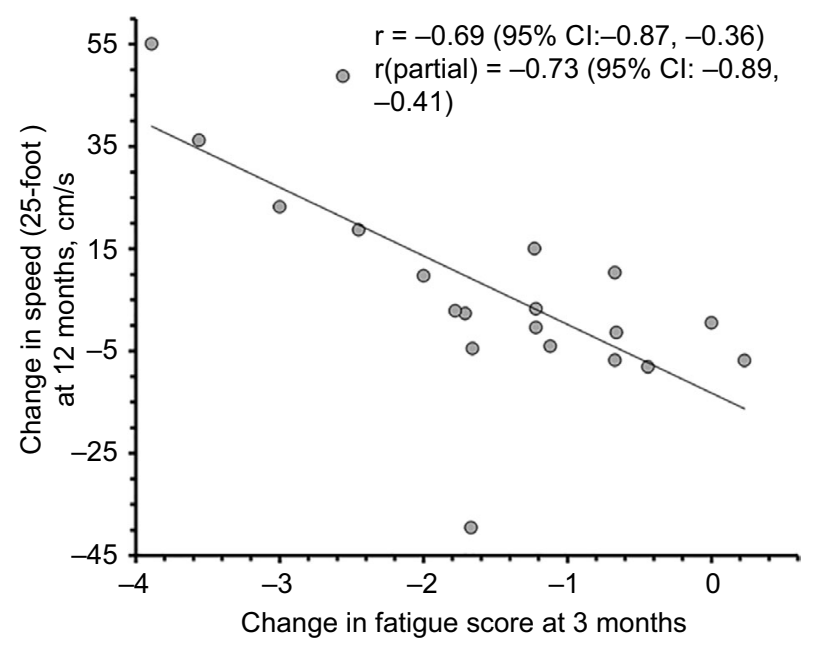

Figure 7 Correlation between changes in fatigue severity scale scores over 3 months and change in 25 -foot walk speed over 12 months.

Notes: $r$, Pearson's correlation coefficient; $r$ (partial), Pearson's partial correlation coefficient after removing the effect of baseline timed 25 -foot walk speed. Abbreviations: $\mathrm{Cl}$, confidence interval.

and static and dynamic balance over 12 months. This suggests that this intervention may be effective in not only slowing the progression but partially reversing gait impairment in some pwMS who are in early stages of the disease and have mildto-moderate gait impairments. In comparison, two Phase III studies investigating the efficacy of dalfampridine (a US FDA-approved drug to improve walking in pwMS) on walking ability of pwMS over a period of 9-14 weeks reported $42.9 \%$ and $35 \%$ responders in the dalfampridine-treated groups and only $8 \%$ and $9.3 \%$ responders, respectively, in the placebo groups. ${ }^{4,5}$ Responders to dalfampridine in these studies showed $\sim 25 \%$ average increase in walking speed during $\mathrm{T} 25 \mathrm{FW}$ over a treatment period of $9-14$ weeks, ${ }^{4,5}$ similar to $\sim 20 \%$ improvement observed at 3 months in TUG-Res to the current intervention. A randomized controlled trial in pwMS reported $10 \%$ increase in median TUG speed from baseline to 6 months following use of prolonged-release fampridine (extended-release dalfampridine in the USA). ${ }^{30}$ Similarly, we observed $11 \%$ decrease in mean time to complete TUG test (thus increase in speed) by the entire cohort and $32.6 \%$ decrease in TUG time of TUG-Res from baseline to 6 months. Notably, the population studied for effects of dalfampridine differed from the current investigation as $\sim 35 \%$ of subjects had RRMS (vs all with progressive MS in the current work) and lower level of disability at baseline (mean EDSS score 5.6-5.8 vs 6.2 in the current study).,5,30 Importantly, no serious side effects leading to discontinuation of the intervention were observed in the current study, whereas $19 \%$ of the subjects in each group (treatment and placebo) discontinued treatment in the prolonged-release fampridine study; adverse effects being the most common reason to discontinue the study. ${ }^{30}$

Interestingly, we did not find any effect of diseasemodifying drug use on changes in TUG and T25FW in our study subjects at 12 months. However, lack of a control group, a small sample size and that only 7 of 20 subjects were using disease-modifying drugs in this study, limits any conclusions regarding concomitant effects of medications in our study subjects. Future studies need to investigate and compare effects of this intervention alone and when used in combination with pharmacological approaches in patients with progressive MS. When use of medications such as dalfampridine is limited due to side effects, beneficial effects of intrathecal application of sustained-release steroid triamcinolone acetonide (TCA) on disability and estimated walking distance have been reported, with no severe side effects in patients with progressive MS. Multiple studies have shown that 6 TCA administrations performed every 3 days within a 3-week period decreased EDSS scores and increased maximum walking distance estimated during EDSS scoring in patients with progressive MS. Unlike current study, these studies did not measure walking speed during standardized tests such as TUG or T25FW. However, significant improvements in EDSS scores of patients with progressive MS within a short period of 3 weeks suggest potential benefits of TCA administration on gait and disability. ${ }^{31-34}$ Since this approach has minimal side effects, it may be used as an adjunct to intervention used in the current study.

Comparison of TUG-Res and TUG-NRes showed that TUG-Res had lesser gait disability at baseline as reflected in their ability to perform parts of TUG test in comparatively shorter time and better spatio-temporal gait parameters than TUG-NRes (Table 4). TUG-NRes needed greater support to walk as more number of subjects were using walker for ambulation compared with TUG-Res, suggesting comparatively greater muscle weakness and poor balance in TUG-NRes. These differences in baseline characteristics of subjects may have affected the response to the intervention. However, 1 subject with very poor walking speed of $6.1 \mathrm{~cm} / \mathrm{s}$ to walk toward 10 feet mark at baseline also showed consistent improvement during the study, to $24.3 \mathrm{~cm} / \mathrm{s}$ at 12 months (Video S1: 00:00:01:04 and 00:00:51:08), suggesting that this intervention can have positive effects in some pwMS with severe disability at baseline. It is noteworthy that this subject had good family support and was able to adhere to the diet $100 \%$ throughout 12 months. Significant differences were not observed over 12 months between TUG-Res and 
TUG-NRes in terms of adherence to the diet and exercise and NMES program, suggesting that baseline characteristics of the subjects may have a greater role than adherence to the multimodal program in terms of their response to the intervention. It is possible that TUG-NRes had greater disease burden than TUG-Res due to which either this intervention was ineffective or dosage of the intervention was insufficient. It is also noteworthy that diet was overall similar for all the subjects but NMES-exercise program were customized for each subject based on his/her ability to perform exercises and assistance available at home. TUG-NRes had comparatively poor walking performance and needed greater support for ambulation suggesting greater muscle weakness than TUGRes. Subjects with severe muscle weakness were able to perform only low-intensity exercises (e.g., isometric contractions) due to inability to generate sufficient torque to move the relevant body segment, whereas subjects with comparatively higher muscle strength could start with concentric muscle contractions to move body segments, which may have led to greater improvement in muscle strength as reflected in subjects' gait and balance tests. Future studies are needed to study dose response to exercise and NMES program in subjects with progressive MS.

We also found that reduction in perceived fatigue of entire cohort over first 3 months following the intervention was strongly associated with increase in walking speed during $\mathrm{T} 25 \mathrm{FW}$ test over 12 months. It is possible that large decreases in fatigue (e.g., by 1.8 or more on the FSS, Figure 7) over the initial 3 months of the intervention facilitated increases in T25FW speed at 12 months because these subjects would be motivated to walk more often and for greater distances in their daily life. Smaller decreases in fatigue may be insufficient to contribute to increased motivation to walk during daily life. It is also noteworthy that we observed highest decrease in fatigue scores in first 3 months (average decrease of 1.5 points), and then continued gradual improvement from 6 to 12 months. Mean 25-foot walk speed of TUG-Res showed a similar pattern of greatest increase in first 3 months and then a steady improvement over the remaining study period. Previously, we reported that high responders to this intervention in terms of fatigue (average $>2$ point decrease in FSS at each time point of the study from baseline) were in better physical health at baseline than low responders. ${ }^{20}$ At baseline, high responders had lower EDSS scores (median: 6 vs $6.5, p<0.0005)$ and higher physical functioning scores on short form-36 (median: 15 vs $5, p=0.031$ ) compared with low responders. ${ }^{20}$ Thus, both our reports suggest that baseline physical health may have significant effects on subjects' responses to this intervention. Initiating this multimodal intervention during earlier stages of the disease may be more effective in reducing progression and, in some cases, be capable of regression of gait and balance impairments due to MS.

Effects of a multimodal intervention similar to that used in the current study on gait and balance of pwMS have not been studied previously. However, as mentioned earlier, benefits of components of this multimodal intervention such as resistance exercise and NMES have been reported. ${ }^{7-9}$ Although those studies were for comparatively short duration and differed in subjects' level of disability and diagnosis, they support our findings. TUG-Res in the current study showed consistent improvements in TUG, T25FW, and Berg Balance Scale scores from baseline to 12 months. Increases in walking speed during TUG were mediated by increased step lengths and decreased step durations. Both shorter and longer step lengths increased in TUG-Res, suggesting that both stronger and weaker lower limbs' muscles responded to the intervention. Although small, the improved Berg Balance Scale scores indicate improved static and dynamic balance. These improvements were possibly due to the combined effects of different components of this intervention on strength and coordination of lower limb muscles during gait and balance tasks.

\section{Limitations}

A small sample size and lack of control group were the main limitations of this study. This was a pilot study designed to assess effects of a multimodal intervention on gait and balance of subjects with progressive MS and collect data for future larger studies. We do not intend to claim efficacy of this intervention in improving symptoms associated with MS. However, it is noteworthy that all the subjects in this study were in the progressive phase and are anticipated to show decline in their gait and balance over 12 months. Our findings that some of the subjects responded well to this interventions are noteworthy and provide useful information about the progressive MS patient population for future studies of this intervention.

\section{Acknowledgments}

We are very thankful to Dr Catherine Swanson, MD, and student researchers who supported this project during data collection: Danielle Klein, Nicole Grogan, John Bolton, Alejandra Gonzalez, Allison Banwort, Alison Crimson, Danielle Dietz, Emily Bradley, Summer Anderson, Glenda Mutinda, Carl Sohocki, and Owen Sessions. 
This work was funded by Direct-MS and in-kind support was provided by the Institute for Clinical and Translational Science at the University of Iowa, which is supported by the National Institutes of Health (NIH) Clinical and Translational Science Award (CTSA) program, grant U54TR001356. The CTSA program is led by the NIH's National Center for Advancing Translational Sciences. This publication's contents are the sole responsibility of the authors and do not necessarily represent the official views of the NIH or the VA. In-kind support was also provided from DJO Inc., Pinnaclife Inc., TZ Press LLC, and the Iowa City VA Healthcare System, Iowa City, IA.

\section{Disclosure}

Dr Terry L Wahls has equity interest in the following companies: Dr Terry Wahls LLC; TZ Press LLC; RDT LLC; and the website www.terrywahls.com. She also owns the copyrights to the books Minding My Mitochondria, 2nd Edition, The Wahls Protocol, and The Wahls Protocol Cooking for Life. Dr Wahls has conflicts of interest management plans in place with both the University of Iowa and the Iowa City VA Healthcare System. All other authors report no conflicts of interest.

\section{References}

1. Hemmett L, Holmes J, Barnes M, Russell N. What drives quality of life in multiple sclerosis? QJM. 2004;97(10):671-676.

2. Kelleher KJ, Spence W, Solomonidis S, Apatsidis D. Ambulatory rehabilitation in multiple sclerosis. Disabil Rehabil. 2009;31(20):1625-1632.

3. Coleman CI, Sidovar MF, Roberts MS, Kohn C. Impact of mobility impairment on indirect costs and health-related quality of life in multiple sclerosis. PLoS One. 2013;8(1):e54756.

4. Goodman AD, Brown TR, Edwards KR, et al. A phase 3 trial of extended release oral dalfampridine in multiple sclerosis. Ann. Neurol. 2010;68(4):494-502.

5. Goodman AD, Brown TR, Krupp LB, et al. Sustained-release oral fampridine in multiple sclerosis: a randomised, double-blind, controlled trial. Lancet. 2009;373(9665):732-738.

6. Cameron MH, Fitzpatrick M, Overs S, Murchison C, Manning J, Whitham R. Dalfampridine improves walking speed, walking endurance, and community participation in veterans with multiple sclerosis: a longitudinal cohort study. Mult Scler. 2014;20(6):733-738.

7. Latimer-Cheung AE, Pilutti LA, Hicks AL, et al. Effects of exercise training on fitness, mobility, fatigue, and health-related quality of life among adults with multiple sclerosis: a systematic review to inform guideline development. Arch Phys Med Rehabil. 2013;94(9): 1800.e3-1828.e3.

8. Motl RW, Pilutti LA. The benefits of exercise training in multiple sclerosis. Nat Rev Neurol. 2012;8(9):487-497.

9. Ratchford JN, Shore W, Hammond ER, et al. A pilot study of functional electrical stimulation cycling in progressive multiple sclerosis. NeuroRehabilitation. 2010;27(2):121-128.

10. Stein RB, Everaert DG, Thompson AK, et al. Long-term therapeutic and orthotic effects of a foot drop stimulator on walking performance in progressive and nonprogressive neurological disorders. Neurorehabil Neural Repair. 2010;24(2):152-167.
11. Barrett CL, Mann GE, Taylor PN, Strike P. A randomized trial to investigate the effects of functional electrical stimulation and therapeutic exercise on walking performance for people with multiple sclerosis. Mult Scler. 2009;15(4):493-504.

12. Miller L, McFadyen A, Lord AC, et al. Functional electrical stimulation for foot drop in multiple sclerosis: a systematic review and metaanalysis of the impact on gait speed. Arch Phys Med Rehabil. Epub 2017 Jan 11.

13. Artemiadis AK, Vervainioti AA, Alexopoulos EC, Rombos A, Anagnostouli MC, Darviri C. Stress management and multiple sclerosis: a randomized controlled trial. Arch Clin Neuropsychol. 2012;27(4): 406-416.

14. Mohr DC, Lovera J, Brown T, et al. A randomized trial of stress management for the prevention of new brain lesions in MS. Neurology. 2012;79(5):412-419.

15. Reese D, Shivapour ET, Wahls TL, Dudley-Javoroski SD, Shields R. Neuromuscular electrical stimulation and dietary interventions to reduce oxidative stress in a secondary progressive multiple sclerosis patient leads to marked gains in function: a case report. Cases J. 2009; 2:7601.

16. Wahls TL, Reese D, Kaplan D, Darling WG. Rehabilitation with neuromuscular electrical stimulation leads to functional gains in ambulation in patients with secondary progressive and primary progressive multiple sclerosis: a case series report. J Altern Complement Med. 2010;16(12):1343-1349.

17. Cohen JA, Cutter GR, Fischer JS, et al. Benefit of interferon beta1a on MSFC progression in secondary progressive MS. Neurology. 2002;59(5):679-687.

18. Vermersch P, Benrabah R, Schmidt N, et al. Masitinib treatment in patients with progressive multiple sclerosis: a randomized pilot study. BMC Neurol. 2012;12:36.

19. Bisht B, Darling WG, Grossmann RE, et al. A multimodal intervention for patients with secondary progressive multiple sclerosis: feasibility and effect on fatigue. J Altern Complement Med. 2014;20(5): 347-355.

20. Bisht B, Darling WG, Shivapour ET, et al. Multimodal intervention improves fatigue and quality of life of subjects with progressive multiple sclerosis: a pilot study. Degenerative Neurological Neuromuscular Disease. 2015;2015(5):19-35.

21. Cutlip RG, Mancinelli C, Huber F, DiPasquale J. Evaluation of an instrumented walkway for measurement of the kinematic parameters of gait. Gait Posture. 2000;12(2):134-138.

22. Krupp LB, LaRocca NG, Muir-Nash J, Steinberg AD. The fatigue severity scale. Application to patients with multiple sclerosis and systemic lupus erythematosus. Arch. Neurol. 1989;46(10):1121-1123.

23. Kapoor R, Furby J, Hayton T, et al. Lamotrigine for neuroprotection in secondary progressive multiple sclerosis: a randomised, doubleblind, placebo-controlled, parallel-group trial. Lancet Neurol. 2010; 9(7):681-688.

24. Khaleeli Z, Sastre-Garriga J, Ciccarelli O, Miller DH, Thompson AJ. Magnetisation transfer ratio in the normal appearing white matter predicts progression of disability over 1 year in early primary progressive multiple sclerosis. J Neurol Neurosurg Psychiatry. 2007;78(10): 1076-1082.

25. Nilsagard Y, Lundholm C, Gunnarsson LG, Dcnison E. Clinical relevance using timed walk tests and 'timed up and go' testing in persons with multiple sclerosis. Physiother Res Int. 2007;12(2): $105-114$.

26. Hobart J, Blight AR, Goodman A, Lynn F, Putzki N. Timed 25-foot walk: direct evidence that improving $20 \%$ or greater is clinically meaningful in MS. Neurology. 2013;80(16):1509-1517.

27. Learmonth YC, Paul L, McFadyen AK, Mattison P, Miller L. Reliability and clinical significance of mobility and balance assessments in multiple sclerosis. Int J Rehabil Res. 2012;35(1):69-74.

28. Larocca NG. Impact of walking impairment in multiple sclerosis: perspectives of patients and care partners. Patient. 2011;4(3):189-201. 
29. Salter AR, Cutter GR, Tyry T, Marrie RA, Vollmer T. Impact of loss of mobility on instrumental activities of daily living and socioeconomic status in patients with MS. Curr Med Res Opin. 2010;26(2):493-500.

30. Hupperts R, Lycke J, Short C, et al. Prolonged-release fampridine and walking and balance in MS: randomised controlled MOBILE trial. Mult Scler. 2016;22(2):212-221.

31. Hoffmann V, Schimrigk S, Islamova S, et al. Efficacy and safety of repeated intrathecal triamcinolone acetonide application in progressive multiple sclerosis patients. J Neurol Sci. 2003;211(1-2):81-84.
32. Hoffmann V, Kuhn W, Schimrigk S, et al. Repeat intrathecal triamcinolone acetonide application is beneficial in progressive MS patients. Eur J Neurol. 2006;13(1):72-76.

33. Hellwig K, Stein FJ, Przuntek H, Muller T. Efficacy of repeated intrathecal triamcinolone acetonide application in progressive multiple sclerosis patients with spinal symptoms. BMC Neurol. 2004;4(1):18.

34. Abu-Mugheisib M, Benecke R, Zettl UK. Repeated intrathecal triamcinolone acetonide administration in progressive multiple sclerosis: a review. MultSclerInt. 2011;2011:219049.
Degenerative Neurological and Neuromuscular Disease

\section{Publish your work in this journal}

Degenerative Neurological and Neuromuscular Disease is an international, peer-reviewed, open access journal focusing on research into degenerative neurological and neuromuscular disease, identification of therapeutic targets and the optimal use of preventative and integrated treatment interventions to achieve improved outcomes, enhanced

\section{Dovepress}

survival and quality of life for the patient. The manuscript management system is completely online and includes a very quick and fair peer-review system. Visit http://www.dovepress.com/testimonials.php to read real quotes from published authors.

Submit your manuscript here: https://www.dovepress.com/degenerative-neurological-and-neuromuscular-disease-journal 